\title{
MULTIPLICATIVE DECOMPOSITION OF PROBABILITY MEASURES
}

\author{
HARALD LUSCHGY
}

(Communicated by R. Daniel Mauldin)

\begin{abstract}
We investigate decompositions of probability measures having the feature that the component of interest in the decomposition of a product probability measure is the product of the corresponding components in the decomposition of the factors.
\end{abstract}

\section{INTRODUCTION}

It is well known that the Lebesgue decomposition is multiplicative in the following sense. Let $P_{i}$ and $Q_{i}$ be probability measures ( $p$-measures) on some $\sigma$ algebra $\mathfrak{U}_{i}$, and let $\mu_{i}$ be the $Q_{i}$-continuous component of $P_{i}$ in the Lebesgue decomposition of $P_{i}$ w.r.t. $Q_{i}, i=1, \ldots, n$. Then the product measure $\otimes_{1}^{n} \mu_{i}$ is the $\otimes_{1}^{n} Q_{i}$-continuous component of $\otimes_{1}^{n} P_{i}$ in the Lebesgue decomposition of $\otimes_{1}^{n} P_{i}$ w.r.t. $\otimes_{1}^{n} Q_{i}$ (cf. [8, p. 54]). This is the origin of the question of which other decompositions share the feature of being multiplicative with the Lebesgue decomposition. In this paper we consider decompositions w.r.t. "band-defining" properties of $p$-measures, such as being monogenic, admitting extensions, being uniquely extensible, and so forth. Observing that, in the above example, $\bigotimes_{1}^{n} \mu_{i}$ is the band projection of $\bigotimes_{1}^{n} P_{i}$ onto the band of $\otimes_{1}^{n} Q_{i}$-continuous, bounded, signed measures, we introduce the notion of a multiplicative band projection and derive a characterization of the band-defining properties which admit a multiplicative band projection. It turns out that exactly those properties are multiplicative that are stable under (direct) products and under forming marginals of measures majorized by a (direct) product (Theorem 2). Several examples are discussed, and a generalization to the setting of invariant measures is given (Theorem 3).

A basic fact. Let us recall a useful characterization of bands of measures. Let $\mathfrak{U}$ be a $\sigma$-algebra of subsets of some set $\Omega$ and let ca(U) denote the order-

Received by the editors November 14, 1989.

1980 Mathematics Subject Classification (1985 Revision). Primary 28A35.

Key words and phrases. Multiplicative band projections, decompositions of product measures, invariant measures.

Research supported by a Heisenberg grant of the Deutsche Forschungsgemeinschaft. 
complete vector lattice of all bounded, signed measures on $\mathfrak{U}$. For a subset $M$ of ca $(\mathfrak{U})$, let $B(M)$ denote the band generated by $M$. For details on bands and related notions see [9].

The following characterization of bands in $\mathrm{ca}(\mathfrak{U})$ is contained in [6] (see [1] for a different proof).

Proposition 1. Let $M \subset \mathrm{ca}(\mathfrak{U})_{+}$. Then $B=\{\mu \in \mathrm{ca}(\mathfrak{U}):|\mu| \in M\}$ is a band in $\mathrm{ca}(\mathfrak{U})$ if and only if $M$ has the following two properties:

(i) $\mu \in M, \nu \in \mathrm{ca}(\mathfrak{U})_{+}, \nu \ll \mu$ imply $\nu \in M$.

(ii) $\mu \in \mathrm{ca}(\mathfrak{U})_{+}, \mu\left(A_{n} \cap \cdot\right) \in M$ for every $n \in \mathbb{N}$, where $\left(A_{n}\right)_{n \in \mathbb{N}}$ is a countable partition of $\Omega$ in $\mathfrak{U}$, imply $\mu \in M$.

Proof. Since $B(\mu)=\{v \in \mathrm{ca}(\mathfrak{U}):|v| \ll \mu\}$ (cf. [9, p. 114]), the "only if" part is clear. The "if" part: By [6, Lemma 2 and Remark], it follows from (i) and (ii) that $B(M)_{+}=M$. This gives $B(M)=B$ and hence, $B$ is a band.

\section{Multiplicative BAND PROJECTIONS}

Let $\mathfrak{U}_{i}$ be a $\sigma$-algebra of subsets of some set $\Omega_{i}$ and let $B_{i}$ denote a band in ca $\left(\mathfrak{U}_{i}\right), i=1, \ldots, n$. Let $B$ be a band in $\operatorname{ca}\left(\otimes_{1}^{n} \mathfrak{U}_{i}\right)$. The band projection $\varphi_{B}$ of $\mathrm{ca}\left(\bigotimes_{1}^{n} \mathfrak{U}_{i}\right)$ onto $B$ is said to be multiplicative w.r.t. $B_{i}$ if

$$
\varphi_{B}\left(\bigotimes_{1}^{n} P_{i}\right)=\bigotimes_{1}^{n} \varphi_{B_{i}}\left(P_{i}\right)
$$

for every $p$-measure $P_{i}$ on $\mathfrak{U}_{i}, i=1, \ldots, n$. Then by the Riesz decomposition theorem (cf. [9, p. 62]), $\otimes_{1}^{n} P_{i}$ has a unique decomposition

$$
\bigotimes_{1}^{n} P_{i}=\bigotimes_{1}^{n} \varphi_{B_{i}}\left(P_{i}\right)+\mu, \quad \text { where } \mu \in B^{\perp} \text {. }
$$

Note that multiplicative band projections satisfy $\varphi_{B}\left(\bigotimes_{1}^{n} \nu_{i}\right)=\bigotimes_{1}^{n} \varphi_{B_{i}}\left(\nu_{i}\right)$ for every $\nu_{i} \in \mathrm{ca}\left(\mathfrak{U}_{i}\right)_{+}$. Concerning applications, the bands $B$ and $B_{1}, \ldots, B_{n}$ will always be related by the same defining property. In the sequel, we deal only with the case $n=2$. Extensions to arbitrary $n$ are obvious.

Define product bands by

$$
B_{1} \tilde{\otimes} B_{2}=B\left(\left\{Q_{1} \otimes Q_{2}: Q_{i} \in B_{i} p \text {-measure, } i=1,2\right\}\right)
$$

and

$$
B_{1} \hat{\otimes} B_{2}=B\left(\left\{Q \in \mathrm{ca}\left(\mathfrak{U}_{1} \otimes \mathfrak{U}_{2}\right): Q p \text {-measure, } \pi_{i}(Q) \in B_{i}, i=1,2\right\}\right),
$$

where $\pi_{i}(Q)$ denotes the $i$ th marginal of $Q$. Using Proposition 1, it is not difficult to check that

$$
B_{1} \hat{\otimes} B_{2}=\left\{\mu \in \mathrm{ca}\left(\mathfrak{U}_{1} \otimes \mathfrak{U}_{2}\right): \pi_{i}(|\mu|) \in B_{i}, \quad i=1,2\right\} .
$$

Obviously, we have $B_{1} \tilde{\otimes} B_{2} \subset B_{1} \hat{\otimes} B_{2}$. To see that, in general, $B_{1} \tilde{\otimes} B_{2} \neq B_{1} \hat{\otimes} B_{2}$, let $\Omega_{1}=\Omega_{2}=[0,1], \mathfrak{U}_{1}=\mathfrak{U}_{2}=\mathfrak{U}$ the Borel $\sigma$-algebra, and let $B_{1}=B_{2}=$ $B(\lambda)$, where $\lambda$ is the Lebesgue measure. Then $B_{1} \tilde{\otimes} B_{2}=B\left(\lambda^{2}\right)$ and, for the $p$ - 
measure $Q$ on $\mathfrak{U}^{2}$ defined by $Q(A)=\int 1_{A}(\omega, \omega) d \lambda(\omega)$, we have $\pi_{i}(Q)=\lambda$, and hence $Q \in B(\lambda) \hat{\otimes} B(\lambda)$, but $Q \notin B\left(\lambda^{2}\right)$.

The bands $B$ having a multiplicative band projection can be characterized in terms of the product bands of $B_{i}$ as follows:

Theorem 2. The following statements are equivalent:

(i) $\varphi_{B}$ is multiplicative w.r.t. $B_{1}$ and $B_{2}$.

(ii) $B_{1} \tilde{\otimes} B_{2} \subset B$ and $B \cap\left[0, P_{1} \otimes P_{2}\right] \subset B_{1} \hat{\otimes} B_{2}$ for every p-measure $P_{i}$ on $\mathfrak{U}_{i}, i=1,2$.

(iii) $B \cap\left[0, P_{1} \otimes P_{2}\right]=B_{1} \tilde{\otimes} B_{2} \cap\left[0, P_{1} \otimes P_{2}\right]$ for every $p$-measure $P_{i}$ on $\mathfrak{U}_{i}$, $i=1,2$.

As a consequence, we obtain that the multiplicative property of band projections is stable under intersections of bands.

Corollary. Let $D_{i}$ and $D$ be bands in ca $\left(\mathfrak{U}_{i}\right)$ and ca $(\mathfrak{U})$, respectively, $i=1,2$. If $\varphi_{D}$ is multiplicative w.r.t. $D_{1}, D_{2}$, and $\varphi_{B}$ is multiplicative w.r.t. $B_{1}, B_{2}$, then $\varphi_{B \cap D}$ is multiplicative w.r.t. $B_{1} \cap D_{1}$ and $B_{2} \cap D_{2}$.

Proof. Immediately from Theorem 2 , in view of the relations

$$
B_{1} \cap D_{1} \tilde{\otimes} B_{2} \cap D_{2} \subset B_{1} \tilde{\otimes} B_{2} \cap D_{1} \tilde{\otimes} D_{2}
$$

and

$$
B_{1} \cap D_{1} \hat{\otimes} B_{2} \cap D_{2}=B_{1} \hat{\otimes} B_{2} \cap D_{1} \hat{\otimes} D_{2} .
$$

The proof of the theorem is based on two lemmas.

Lemma 1. If $B_{1} \tilde{\otimes} B_{2} \subset B \subset B_{1} \hat{\otimes} B_{2}$, then $\varphi_{B}$ is multiplicative w.r.t. $B_{1}$ and $B_{2}$.

Proof. Let $P_{i}$ be a $p$-measure on $\mathfrak{U}_{i}, i=1,2$. Since $B_{1} \tilde{\otimes} B_{2} \subset B$, we have $\varphi_{B_{1}}\left(P_{1}\right) \otimes \varphi_{B_{2}}\left(P_{2}\right) \in B$. Let $Q \in B$ be a $p$-measure. Then $\pi_{i}(Q) \in B_{i}, i=1,2$, because $B \subset B_{1} \hat{\otimes} B_{2}$. Thus $P_{i}-\varphi_{B_{i}}\left(P_{i}\right) \perp \pi_{i}(Q)$, and so there exists $A_{i} \in \mathfrak{U}_{i}$ such that $P_{i}\left(A_{i}\right)-\varphi_{B_{i}}\left(P_{i}\right)\left(A_{i}\right)=0$ and $\pi_{i}(Q)\left(A_{i}\right)=1$. For $A=A_{1} \times A_{2}$, we obtain

$$
P_{1} \otimes P_{2}(A)-\varphi_{B_{1}}\left(P_{1}\right) \otimes \varphi_{B_{2}}\left(P_{2}\right)(A)=0
$$

and

$$
Q(A)=Q\left(A_{1} \times \Omega_{2} \cap \Omega_{1} \times A_{2}\right)=1 .
$$

This yields $P_{1} \otimes P_{2}-\varphi_{B_{1}}\left(P_{1}\right) \otimes \varphi_{B_{2}}\left(P_{2}\right) \perp Q$. Hence, $Q$ being arbitrary, $P_{1} \otimes P_{2}-$ $\varphi_{B_{1}}\left(P_{1}\right) \otimes \varphi_{B_{2}}\left(P_{2}\right) \in B^{\perp}$. This establishes $\varphi_{B}\left(P_{1} \otimes P_{2}\right)=\varphi_{B_{1}}\left(P_{1}\right) \otimes \varphi_{B_{2}}\left(P_{2}\right)$.

From the preceding lemma one can deduce that the band projections of the product bands coincide when applied to product measures.

Lemma 2. $B_{1} \tilde{\otimes} B_{2} \cap\left[0, P_{1} \otimes P_{2}\right]=B_{1} \hat{\otimes} B_{2} \cap\left[0, P_{1} \otimes P_{2}\right]$ for every p-measure $P_{i}$ on $\mathfrak{U}_{i}, i=1,2$.

Proof. For $\mu \in B_{1} \hat{\otimes} B_{2}, 0 \leq \mu \leq P_{1} \otimes P_{2}$, we have by Lemma 1

$$
\mu \leq \varphi_{B_{1}} \widehat{\bigotimes}_{B_{2}}\left(P_{1} \otimes P_{2}\right)=\varphi_{B_{1}}\left(P_{1}\right) \otimes \varphi_{B_{2}}\left(P_{2}\right) \text {. }
$$


Since the latter product measure belongs to $B_{1} \tilde{\otimes} B_{2}$, we get $\mu \in B_{1} \tilde{\otimes} B_{2}$. Proof of Theorem 2. (i) $\Rightarrow$ (ii). For $p$-measures $Q_{i} \in B_{i}$, we have $\varphi_{B}\left(Q_{1} \otimes Q_{2}\right)=\varphi_{B_{1}}\left(Q_{1}\right) \otimes \varphi_{B_{2}}\left(Q_{2}\right)=Q_{1} \otimes Q_{2}$ and hence, $Q_{1} \otimes Q_{2} \in B$. This yields $B_{1} \tilde{\otimes} B_{2} \subset B$. Moreover, for $\mu \in B$ satisfying $0 \leq \mu \leq P_{1} \otimes P_{2}$ for some $p$-measures $P_{i}$ on $\mathfrak{U}_{i}$, we obtain $\mu \leq \varphi_{B}\left(P_{1} \otimes P_{2}\right)=\varphi_{B_{1}}\left(P_{1}\right) \otimes \varphi_{B_{2}}\left(P_{2}\right)$. Since the latter product measure belongs to $B_{1} \tilde{\otimes} B_{2} \subset B_{1} \hat{\otimes} B_{2}$, it follows that $\mu \in B_{1} \hat{\otimes} B_{2}$.

(ii) $\Rightarrow$ (iii) is an immediate consequence of Lemma 2 .

(iii) $\Rightarrow$ (i). Let $P_{i}$ be a $p$-measure on $\mathfrak{U}_{i}, i=1,2$. Then

$$
\begin{aligned}
\varphi_{B}\left(P_{1} \otimes P_{2}\right) & =\sup B \cap\left[0, P_{1} \otimes P_{2}\right]=\sup B_{1} \tilde{\otimes} B_{2} \cap\left[0, P_{1} \otimes P_{2}\right] \\
& =\varphi_{B_{1}} \widetilde{\bigotimes}_{B_{2}}\left(P_{1} \otimes P_{2}\right)=\varphi_{B_{1}}\left(P_{1}\right) \otimes \varphi_{B_{2}}\left(P_{2}\right) .
\end{aligned}
$$

where the last equation follows from Lemma 1.

\section{EXAMPLes}

3.1. Generalized Lebesgue decomposition. Let $\mathfrak{Q}_{i}$ be a set of $p$-measures on $\mathfrak{U}_{i}$, $i=1,2$, and let $\mathfrak{Q}_{1} \otimes \mathfrak{Q}_{2}=\left\{Q_{1} \otimes Q_{2}: Q_{i} \in \mathfrak{Q}_{i}, i=1,2\right\}$. We claim that

$$
B\left(\mathfrak{Q}_{1} \otimes \mathfrak{Q}_{2}\right)=B\left(\mathfrak{Q}_{1}\right) \tilde{\otimes} B\left(\mathfrak{Q}_{2}\right) \text {. }
$$

The inclusion $\subset$ is obvious. To prove the converse inclusion, note that $\mu$ belongs to $B(\mathfrak{Q})$ if and only if $|\mu| \ll \mathfrak{Q}_{0}\left(Q(A)=0\right.$ for every $Q \in \mathfrak{Q}_{0}$ implies $|\mu|(A)=0)$ for some countable subset $\mathfrak{Q}_{0}$ of $\mathfrak{Q}$. This follows from Proposition 1. Now let $P_{i} \in B\left(\mathfrak{Q}_{i}\right)$ be a $p$-measure, and choose a countable subset $\mathfrak{Q}_{i 0}$ of $\mathfrak{Q}_{i}$ such that $P_{i} \ll \mathfrak{Q}_{i 0}, i=1,2$. Then $P_{1} \otimes P_{2} \ll \mathfrak{Q}_{10} \otimes \mathfrak{Q}_{20}$, hence $P_{1} \otimes P_{2} \in B\left(\mathfrak{Q}_{1} \otimes \mathfrak{Q}_{2}\right)$, and our claim is proved. It follows from Theorem 2 (or Lemma 1) that $\varphi_{B\left(\mathfrak{Q}_{1} \otimes \mathfrak{Q}_{2}\right)}$ is multiplicative w.r.t. $B\left(\mathfrak{Q}_{1}\right)$ and $B\left(\mathfrak{Q}_{2}\right)$. In particular, $\varphi_{B\left(Q_{1} \otimes Q_{2}\right)}$ is multiplicative w.r.t. $B\left(Q_{1}\right)$ and $B\left(Q_{2}\right)$ for every $p$-measure $Q_{i}$ on $\mathfrak{U}_{i}$. This re-establishes the known fact that the Lebesgue decomposition is multiplicative.

3.2. A variant of 3.1 is as follows. Let $B_{i}=\left\{\nu \in \mathrm{ca}\left(\mathfrak{U}_{i}\right):|\nu| \ll \mathfrak{Q}_{i}\right\}$, and $B=\left\{\mu \in \mathrm{ca}\left(\mathfrak{U}_{1} \otimes \mathfrak{U}_{2}\right):|\mu| \ll \mathfrak{Q}_{1} \otimes \mathfrak{Q}_{2}\right\}$. By Proposition $1, B$ and $B_{i}$ are bands. Here we obviously have

$$
B_{1} \tilde{\otimes} B_{2} \subset B \subset B_{1} \hat{\otimes} B_{2} .
$$

Thus $\varphi_{B}$ is multiplicative w.r.t. $B_{1}$ and $B_{2}$.

3.3. Monogenic measures. Let $\mathfrak{B}_{i}$ be a sub- $\sigma$-algebra of $\mathfrak{U}_{i}, i=1,2$. A measure $\nu \in \mathrm{ca}\left(\mathfrak{U}_{i}\right)_{+}$is said to be $\mathfrak{B}_{i}$-monogenic if $\lambda \in \mathrm{ca}\left(\mathfrak{U}_{i}\right)_{+}, \lambda\left|\mathfrak{B}_{i}=\nu\right| \mathfrak{B}_{i}$ imply $\lambda=\nu$. In other words, the set $E\left(\nu \mid \mathfrak{B}_{i}, \mathfrak{U}_{i}\right)=\left\{\lambda \in \operatorname{ca}\left(\mathfrak{U}_{i}\right)_{+}: \lambda \mid \mathfrak{B}_{i}=\right.$ $\left.\nu \mid \mathfrak{B}_{i}\right\}$ of all measure extensions of $\nu \mid \mathfrak{B}_{i}$ to $\mathfrak{U}_{i}$ coincides with $\{\nu\}$. Let $B_{i}=$ $\left\{\nu \in \mathrm{ca}\left(\mathfrak{U}_{i}\right):|\nu|\right.$ is $\mathfrak{B}_{i}$-monogenic $\}$ and $B=\left\{\mu \in \mathrm{ca}\left(\mathfrak{U}_{1} \otimes \mathfrak{U}_{2}\right):|\mu|\right.$ is $\mathfrak{B}_{1} \otimes$ $\mathfrak{B}_{2}$-monogenic $\}$. By [3], $B$ and $B_{i}$ are bands, $B_{1} \hat{\otimes} B_{2} \subset B$ and, in general, $B_{1} \hat{\otimes} B_{2} \neq B$. However, we have

$$
B \cap\left[0, P_{1} \otimes P_{2}\right] \subset B_{1} \hat{\otimes} B_{2}
$$


for every $p$-measure $P_{i}$ on $\mathfrak{U}_{i}, i=1,2$. This is a consequence of the following lemma. Thus, by Theorem 2, being monogenic is a multiplicative property.

Lemma 3. Suppose $\mu \in \mathrm{ca}\left(\mathfrak{U}_{1} \otimes \mathfrak{U}_{2}\right)_{+}$satisfies $\mu \ll P_{1} \otimes P_{2}$ for some $p$ measures $P_{i}$ on $\mathfrak{U}_{i}$. Then for $i=1,2,\left\{\pi_{i}(\rho): \rho \in E\left(\mu \mid \mathfrak{B}_{1} \otimes \mathfrak{B}_{2}, \mathfrak{U}_{1} \otimes \mathfrak{U}_{2}\right)\right\}=$ $E\left(\pi_{i}(\mu) \mid \mathfrak{B}_{i}, \mathfrak{U}_{i}\right)$.

Proof. The inclusion $\subset$ is clear. To prove the converse inclusion, let $f$ denote the Radon-Nikodym derivative of $\mu \mid \mathfrak{B}_{1} \otimes \mathfrak{B}_{2}$ w.r.t. $P_{1} \otimes P_{2} \mid \mathfrak{B}_{1} \otimes \mathfrak{B}_{2}$. Then $g=\int f\left(\cdot, \omega_{2}\right) d P_{2}\left(\omega_{2}\right)$ is the Radon-Nikodym derivative of $\pi_{1}(\mu) \mid \mathfrak{B}_{1}$ w.r.t. $P_{1} \mid \mathfrak{B}_{1}$. Let $N=\{g=0\}$, and define a Markov kernel $K$ from $\left(\Omega_{1}, \mathfrak{U}_{1}\right)$ to $\left(\Omega_{2}, \mathfrak{U}_{2}\right)$ by

if $\omega_{1} \notin N$ and

$$
K\left(\omega_{1}, A_{2}\right)=\frac{A_{2}^{\int f\left(\omega_{1}, \omega_{2}\right) d P_{2}\left(\omega_{2}\right)}}{g\left(\omega_{1}\right)}
$$

$$
K\left(\omega_{1}, A_{2}\right)=Q\left(A_{2}\right)
$$

otherwise, where $Q$ is an arbitrary $p$-measure on $\mathfrak{U}_{2}$. Then $N \in \mathfrak{B}_{1}, \pi_{1}(\mu)(N)$ $=0$ and for the generalized product measure $\pi_{1}(\mu) \otimes K \in \mathrm{ca}\left(\mathfrak{U}_{1} \otimes \mathfrak{U}_{2}\right)_{+}$given by

$$
\pi_{1}(\mu) \otimes K\left(A_{1} \times A_{2}\right)=\int_{A_{1}} K\left(\omega_{1}, A_{2}\right) d \pi_{1}(\mu)\left(\omega_{1}\right),
$$

we have $\pi_{1}(\mu) \otimes K\left|\mathfrak{B}_{1} \otimes \mathfrak{B}_{2}=\mu\right| \mathfrak{B}_{1} \otimes \mathfrak{B}_{2}$. Now let $\lambda \in E\left(\pi_{1}(\mu) \mid \mathfrak{B}_{1}, \mathfrak{U}_{1}\right)$, and let $\rho=\lambda \otimes K$. Then $\pi_{1}(\rho)=\lambda$ and since $K\left(\cdot, B_{2}\right)$ is $\mathfrak{B}_{1}$-measurable for every $B_{2} \in \mathfrak{B}_{2}$, we obtain $\rho\left|\mathfrak{B}_{1} \otimes \mathfrak{B}_{2}=\pi_{1}(\mu) \otimes K\right| \mathfrak{B}_{1} \otimes \mathfrak{B}_{2}=\mu \mid \mathfrak{B}_{1} \otimes \mathfrak{B}_{2}$. This completes the proof (for $i=1$ ).

3.4. Extensible measures. Let $\mathfrak{C}_{i}$ be a $\sigma$-algebra on $\Omega_{i}$ with $\mathfrak{U}_{i} \subset \mathfrak{C}_{i}, i=1,2$. Let $B_{i}=\left\{\nu \in \mathrm{ca}\left(\mathfrak{U}_{i}\right): E\left(|\nu|, \mathfrak{C}_{i}\right) \neq \varnothing\right\}$ and let $B=\left\{\mu \in \mathrm{ca}\left(\mathfrak{U}_{1} \otimes \mathfrak{U}_{2}\right)\right.$ : $\left.E\left(|\mu|, \mathfrak{C}_{1} \otimes \mathfrak{C}_{2}\right) \neq \varnothing\right\}$. By [4], [5], $B$ and $B_{i}$ are bands (which is also an easy consequence of Proposition 1; cf. [1]), and clearly

$$
B_{1} \tilde{\otimes} B_{2} \subset B \subset B_{1} \hat{\otimes} B_{2} \text {. }
$$

Thus the property of admitting a measure extension is a multiplicative property.

3.5. Unique measure extensions. In the situation of 3.4 , let $B_{i}=\left\{\nu \in \mathrm{ca}\left(\mathfrak{U}_{i}\right)\right.$ : $\left.\# E\left(|\nu|, \mathfrak{C}_{i}\right)=1\right\}$ and $B=\left\{\mu \in \mathrm{ca}\left(\mathfrak{U}_{1} \otimes \mathfrak{U}_{2}\right): \# E\left(|\mu|, \mathfrak{C}_{1} \otimes \mathfrak{C}_{2}\right)=1\right\}$. By [4], $B$ and $B_{i}$ are bands (cf. also [1]). From 3.3 it follows that $B_{1} \tilde{\otimes} B_{2} \subset B$. Note that, in general, $B \not \subset B_{1} \hat{\otimes} B_{2}$ (cf. [3]). The following lemma shows that

$$
B \cap\left[0, P_{1} \otimes P_{2}\right] \subset B_{1} \hat{\otimes} B_{2}
$$

for every $p$-measure $P_{i}$ on $\mathfrak{U}_{i}$ and therefore, unique extensibility of measures is a multiplicative property.

Lemma 4. Suppose $\mu \in \mathrm{ca}\left(\mathfrak{U}_{1} \otimes \mathfrak{U}_{2}\right)_{+}$satisfies $E\left(\mu, \mathfrak{C}_{1} \otimes \mathfrak{C}_{2}\right) \neq \varnothing$ and $\mu \leq P_{1} \otimes P_{2}$ for some p-measures $P_{i}$ on $\mathfrak{U}_{i}$. Then for $i=1,2,\left\{\pi_{i}(\rho): \rho \in E\left(\mu, \mathfrak{C}_{1} \otimes \mathfrak{C}_{2}\right)\right\}=$ $E\left(\pi_{i}(\mu), \mathfrak{C}_{i}\right)$. 
Proof. We claim that there exists an extension of $\mu$ to $\mathfrak{C}_{1} \otimes \mathfrak{C}_{2}$ which is majorized by a (direct) product measure. By 3.4 , extensibility is a multiplicative property. Therefore, the band component of $P_{1} \otimes P_{2}$ w.r.t. this property is a product measure $\nu_{1} \otimes \nu_{2}, \nu_{i} \in \mathrm{ca}\left(\mathfrak{U}_{i}\right)_{+}$. From the assumptions follow $\mu \leq \nu_{1} \otimes \nu_{2}$. Let $f$ denote the Radon-Nikodym derivative of $\mu$ w.r.t. $\nu_{1} \otimes \nu_{2}$, and let $\lambda_{i} \in E\left(\nu_{i}, \mathfrak{C}_{i}\right)$. Define $\rho \in \mathrm{ca}\left(\mathfrak{C}_{1} \otimes \mathfrak{C}_{2}\right)_{+}$by $\rho(C)=\int_{C} f d \lambda_{1} \otimes \lambda_{2}$. Then $\rho \in E\left(\mu, \mathfrak{C}_{1} \otimes \mathfrak{C}_{2}\right)$, and $\rho \leq \lambda_{1} \otimes \lambda_{2}$. Our claim is proved. Now the assertion follows from Lemma 3.

\subsection{Extreme extensions. In the situation of 3.4, let}

$$
B_{i}=\left\{\nu \in \operatorname{ca}(\mathfrak{U})_{i}: \operatorname{ex} E\left(|\nu|, \mathfrak{C}_{i}\right) \neq \varnothing\right\}, \quad \text { where ex } E\left(|\nu|, \mathfrak{C}_{i}\right)
$$

denotes the set of all extreme points of the convex set $E\left(|\nu|, \mathfrak{C}_{i}\right)$. This set is characterized by the "Douglas criterion" (cf. [7]): $\lambda \in E\left(|\nu|, \mathfrak{C}_{i}\right)$ is an extreme point if and only if, for each $C \in \mathfrak{C}_{i}$ there exists $A \in \mathfrak{U}_{i}$ such that $\lambda(C \Delta A)=0$. Let $B=\left\{\mu \in \mathrm{ca}\left(\mathfrak{U}_{1} \otimes \mathfrak{U}_{2}\right): \operatorname{ex} E\left(|\mu|, \mathfrak{C}_{1} \otimes \mathfrak{C}_{2}\right) \neq \varnothing\right\}$. By [6], $B$ and $B_{i}$ are bands. We have

$$
B_{1} \tilde{\otimes} B_{2} \subset B \text {. }
$$

In fact, let $Q_{i} \in B_{i}$ be $p$-measures, $P_{i} \in \operatorname{ex} E\left(Q_{i}, \mathfrak{C}_{i}\right)$, and $C_{i} \in \mathfrak{C}_{i}, i=1,2$. By the Douglas criterion, one can find sets $A_{i} \in \mathfrak{U}_{i}$ such that $P_{i}\left(C_{i} \Delta A_{i}\right)=0$. Then

$$
C_{1} \times C_{2} \Delta A_{1} \times A_{2} \subset C_{1} \Delta A_{1} \times \Omega_{2} \cup \Omega_{1} \times C_{2} \Delta A_{2}
$$

and hence, $P_{1} \otimes P_{2}\left(C_{1} \times C_{2} \Delta A_{1} \times A_{2}\right)=0$. Again by the Douglas criterion, this gives $P_{1} \otimes P_{2} \in \operatorname{ex} E\left(Q_{1} \otimes Q_{2}, \mathfrak{C}_{1} \otimes \mathfrak{C}_{2}\right)$ because $\left\{C \in \mathfrak{C}_{1} \otimes \mathfrak{C}_{2}\right.$ : there exists $A \in \mathfrak{U}_{1} \otimes \mathfrak{U}_{2}$ with $\left.P(C \Delta A)=0\right\}$ is a $\sigma$-algebra for every $p$-measure $P$ on $\mathfrak{C}_{1} \otimes \mathfrak{C}_{2}$. Thus $Q_{1} \otimes Q_{2} \in B$.

However, $Q_{1} \otimes Q_{2} \in B$ does not imply $Q_{i} \in B_{i}, i=1,2$. Thus, by Theorem 2 , existence of an extreme extension is not a multiplicative property. To see this, let $\Omega_{1}=\Omega_{2}=[0,1], \mathfrak{U}_{1}=\mathfrak{C}_{1}=\mathfrak{C}_{2}=\mathfrak{C}$ (the Borel $\sigma$-algebra), and $\mathfrak{U}_{2}=\mathfrak{U}$ (the $\sigma$-algebra consisting of all countable sets and their complements). Let $Q=\lambda \otimes$ $\lambda \mid \mathfrak{U}$, where $\lambda$ denotes the Lebesgue measure. Clearly ex $E(\lambda \mid \mathfrak{U}, \mathfrak{C})=\varnothing$. Define a $p$-measure $P$ on $\mathfrak{C}^{2}$ by $P(C)=\int 1_{C}(\omega, \omega) d \lambda(\omega)$. Then $P \mid \mathfrak{C} \otimes \mathfrak{U}=Q$. Let $C_{i} \in \mathfrak{C}, i=1,2$, and let $A=C_{1} \cap C_{2} \times[0,1]$. Since $C_{1} \times C_{2} \Delta A$ is contained in the complement of the diagonal and $P$ is concentrated on the diagonal, $P\left(C_{1} \times C_{2} \Delta A\right)=0$. From the Douglas criterion it follows that $P \in \operatorname{ex} E\left(Q, \mathfrak{C}^{2}\right)$.

\section{INVARIANT MEASURES}

In this section, we establish a generalization of Theorem 2 to invariant measures. Let $G_{i}$ be a semigroup acting on $\Omega_{i}$ (from the left) and assume that $\mathfrak{U}_{i}$ is a $G_{i}$-invariant $\sigma$-algebra; i.e., $g^{-1} A=\left\{\omega \in \Omega_{i}: g \omega \in A\right\} \in \mathfrak{U}_{i}$ for every $g \in G_{i}, A \in \mathfrak{U}_{i}, i=1,2$. Then $G_{i}$ acts on ca $\left(\mathfrak{U}_{i}\right)$ by $g \nu(A)=\nu\left(g^{-1} A\right)$. Let $\mathrm{ca}\left(\mathfrak{U}_{i}\right)_{G_{i}}=\left\{\nu \in \mathrm{ca}\left(\mathfrak{U}_{i}\right): g \nu=\nu\right.$ for every $\left.g \in G_{i}\right\}$ be the order-complete vector sublattice of $\mathrm{ca}\left(\mathfrak{U}_{i}\right)$ consisting of $G_{i}$-invariant elements (cf. [4]). Furthermore, 
let $G$ be a semigroup acting on $\Omega_{1} \times \Omega_{2}$ such that $\mathfrak{U}_{1} \otimes \mathfrak{U}_{2}$ is $G$-invariant. To relate the semigroup actions, make the following assumptions:

(C.1) If $\nu_{i} \in \mathrm{ca}\left(\mathfrak{U}_{i}\right)_{+}$is $G_{i}$-invariant, $i=1,2$, then $\nu_{1} \otimes \nu_{2}$ is $G$-invariant.

(C.2) If $\mu \in \mathrm{ca}\left(\mathfrak{U}_{1} \otimes \mathfrak{U}_{2}\right)_{+}$is $G$-invariant, then $\pi_{i}(\mu)$ is $G_{i}$-invariant, $i=$ 1,2 .

For instance, if $G=G_{1} \times G_{2}$, the direct product of $G_{1}$ and $G_{2}$, acting on $\Omega_{1} \times \Omega_{2}$ by $\left(g_{1}, g_{2}\right)\left(\omega_{1}, \omega_{2}\right)=\left(g_{1} \omega_{1}, g_{2} \omega_{2}\right)$, then $\mathfrak{U}_{1} \otimes \mathfrak{U}_{2}$ is $G$-invariant and (C.1), (C.2) are satisfied. Also, if $G_{1}=G_{2}=G$, where $G$ acts componentwise on $\Omega_{1} \times \Omega_{2}$, then the above conditions are satisfied.

Let $B_{i}$ be a band in ca $\left(\mathfrak{U}_{i}\right)_{G_{i}}$, and let $B$ be a band in ca $\left(\mathfrak{U}_{1} \otimes \mathfrak{U}_{2}\right)_{G}$. The band projection $\varphi_{B, G}$ of $\mathrm{ca}\left(\mathfrak{U}_{1} \otimes \mathfrak{U}_{2}\right)_{G}$ onto $B$ is said to be multiplicative w.r.t. $B_{1}$ and $B_{2}$ if

$$
\varphi_{B, G}\left(P_{1} \otimes P_{2}\right)=\varphi_{B_{1}, G_{1}}\left(P_{1}\right) \otimes \varphi_{B_{2}, G_{2}}\left(P_{2}\right)
$$

for every $G_{i}$-invariant $p$-measure $P_{i}$ on $\mathfrak{U}_{i}, i=1,2$. Here we use (C.1). The product bands of $B_{1}$ and $B_{2}$ in ca $\left(\mathfrak{U}_{1} \otimes \mathfrak{U}_{2}\right)_{G}$ are defined as in $\S 2$ with band generation in ca $\left(\mathfrak{U}_{1} \otimes \mathfrak{U}_{2}\right)_{G}$ and are denoted by $B_{1} \widetilde{\otimes}_{G} B_{2}$ and $B_{1} \widehat{\otimes}_{G} B_{2}$. Using (C.2) and, e.g., [6, Lemma 2], one can easily see that

$$
B_{1} \widehat{\bigotimes}_{G} B_{2}=\left\{\mu \in \mathrm{ca}\left(\mathfrak{U}_{1} \otimes \mathfrak{U}_{2}\right)_{G}: \pi_{i}(|\mu|) \in B_{i}, i=1,2\right\} .
$$

Analogous to Theorem 2 we have the following:

Theorem 3. Suppose (C.1) and (C.2) are satisfied. Then the following statements are equivalent

(i) $\varphi_{B, G}$ is multiplicative w.r.t. $B_{1}$ and $B_{2}$.

(ii) $B_{1} \widetilde{\otimes}_{G} B_{2} \subset B$ and $B \cap\left[0, P_{1} \otimes P_{2}\right]_{G} \subset B_{1} \widehat{\otimes}_{G} B_{2}$ for every $G_{i}$-invariant p-measure $P_{i}$ on $\mathfrak{U}_{i}, i=1,2\left(\left[0, P_{1} \otimes P_{2}\right]_{G}\right.$ is the order interval in $\left.\mathrm{ca}\left(\mathfrak{U}_{1} \otimes \mathfrak{U}_{2}\right)_{G}\right)$.

(iii) $B \cap\left[0, P_{1} \otimes P_{2}\right]_{G}=B_{1} \widetilde{\otimes}_{G} B_{2} \cap\left[0, P_{1} \otimes P_{2}\right]_{G}$.

Combining this theorem with results in [2], [4], and [5], one may check that under suitable assumptions on the semigroups, invariant versions of the examples given in $\S 3$ are valid.

\section{ACKNOWLEDGMENT}

I would like to thank to D. Plachky, who brought this topic to my attention.

\section{REFERENCES}

1. W. Hackenbroch, On band-defining properties in measure extension problems, preprint, 1989.

2. __ Bands of invariantly extensible measures, Manuscripta Math. 66 (1990), 351-358.

3. Z. Lipecki and D. Plachky, On monogenic operators and measures, Proc. Amer. Math. Soc. 82 (1981), 216-220. 
4. H. Luschgy, Invariant extensions of positive operators and extreme points, Math. Z. 171 (1980), 75-81.

5. __ Extreme invariant extensions of probability measures and probability contents, Illinois J. Math. 26 (1982), 27-40.

6. __ Decomposition of charges and measures. Proc. Amer. Math. Soc. 96 (1986), 121-125.

7. D. Plachky, Extremal and monogenic additive set functions. Proc. Amer. Math. Soc. $\mathbf{5 4}$ (1976), 193-196.

8. __ Stochastik-Anwendungen und Ubungen, Akademische Verlagsgesellschaft, Wiesbaden, 1983.

9. H. H. Schaefer, Banach lattices and positive operators, Springer, Berlin, 1974.

Universität Münster, Institut für Mathematische Statistik, Einsteinstrasse 62, D4400 Münster, Federal Republic of Germany 